Original article

Paediatrics Today 2016;12(1):149-159

DOI $10.5457 / \mathrm{p} 2005-114.151$

\title{
ULTRASOUND-GUIDED REDUCTION OF ILEOCOLIC INTUSSUSCEPTION IN CHILDREN
}

\author{
Ana TRIPALO BATOŠ, Tonći GRMOJA, Goran ROIĆ
}

Department for pediatric radiology

Children's Hospital, Zagreb, Croatia

\author{
Correspondence: \\ abatosh@gmail.com \\ Tel.: + 385914600289 \\ Fax.: + 38514600113
}

Received: February 23, 2016

Accepted: May 16, 2016

Key words: Hydrostatic reduction Intussusception - Pediatric ultrasound.

\begin{abstract}
Objective - To determine the success rate of the newly introduced method of ultrasound guided hydrostatic reduction in detected ileocolic intussusception by retrospective analysis. Materials and methods - Analysis was performed on all diagnosed ileocolic intussusceptions during the period of December 2013 to November 2015 at the Department for pediatric Radiology of the Children's Hospital Zagreb. In this period of time 34 patients were diagnosed with ileocolic intussusception. Ultrasound guided hydrostatic reduction was performed in 31 patients. We performed ultrasound guided hydrostatic reposition using saline solution warmed at body temperature. Saline was applied per rectum. When the intussusceptum crossed the ileocecal valve, the reposition was considered successful. Results - Out of 34 patients, three patients were treated with an urgent surgical procedure, while ultrasound guided hydrostatic reduction was performed in 31 patients. The non-surgical procedures were successful in all cases and complete reductions of intussusception were achieved in all 31 patients. In three patients, reinvagination occurred within the first 48 hours. Conclusion - Ultrasound guided hydrostatic reduction of ileocolic intussusceptions in children is a very simple and effective technique. The success rate was $91.2 \%(31 / 34)$. The recurrence rate was $8.8 \%$.
\end{abstract}

\section{Introduction}

An intussusception is defined as the invagination of a segment of the proximal bowel (intussusceptum) into the lumen of the distal bowel (intussuscipiens). The incidence of intussusception is 2 per 1,000 children (1). Ileocolic intussusception is a common cause of childhood abdominal pain and intestinal obstruction and therefore a common surgical emergency in the pediatric population. The peak incidence is in children from 6 months up to 2 years of age (2), where enlarged mesenteric lymph nodes act as a "leading point", mostly due to acute abdominal infection (3,
4). In children younger than 6 months and older than 2 years, associated bowel abnormality should be considered as intussusceptum (Meckel diverticulum, duplication cyst, ectopic pancreas, and tumors) (5). Intussusception is more common in children who have undergone recent abdominal surgery (6).

When diagnosed early, with appropriate fluid reduction and treatment, the mortality rate from intussusception in children is less than $1 \%$. If left untreated, this condition is uniformly fatal within 2 to 5 days (5). Ultrasound is the method of choice for the detection of intussusception, which may demon- 
strate typical appearances. After detecting an ileocolic intussusception, the choice of reduction can be either nonsurgical or surgical (7). Increased caution is needed if the symptoms are long-standing ( $>2-3$ days) or if there are signs of peritonitis $(8,9)$. Patients with pneumoperitoneum, peritonitis or shock should be treated with immediate surgery $(3,10)$.

The management of intussusception remains dependent on the local experience and practices of the radiology and surgery departments, although more recently there has been a significant positive trend toward enema reduction (11). At our institution we rely on ultrasound as the primary diagnostic tool for suspected intussusception. Ultrasound is a highly sensitive and specific test for intussusception and is the imaging modality of choice (12). Intussusception can easily be identified even by inexperienced radiologists (3).

When diagnosed, the initial treatment of choice for intussusception is nonsurgical reduction with an air or hydrostatic enema. We use ultrasound guided hydrostatic reduction with warm saline solution at body temperature. The main contraindication is perforation (13). Ultrasound-guided reduction of ileocolic intussusception was first described in 1982 by Kim et al. and then extensively used in clinical services (14). There are different techniques, depending on experience and previously used methods for reduction of intussusception.

In our study, we retrospectively analyzed the success rate of ultrasound guided hydrostatic reduction in patients with detected ileocolic invagination, which was introduced recently at our department.

\section{Materials and methods}

Our retrospective study was approved by the Ethics Committee of the Children's Hospital Zagreb. Retrospective analysis of patients' records and electronic databases was per- formed. In the period of time from December 2013 to November 2015, a total number of 34 patients with ileocolic intussusception were diagnosed at the Department for pediatric Radiology of the Children's Hospital Zagreb, Croatia. In three patients reinvagination was confirmed.

All ultrasound scans were performed on the GE Logiq S8 machine, starting with a conventional abdominal ultrasound scan with a convex transducer ( 3 to $7,5 \mathrm{MHz}$ ) dedicated to children. The scan was continued using a high-frequency linear probe in a transverse orientation to the bowel (5-12 $\mathrm{MHz}$ ) from the level of cecum, tracing the colon forward to the rectum. The majority of intussusceptions was ileocolic and positioned in the right subhepatic region. On a transverse view, the alternating concentric hypoechoic and echogenic layers of an intussusception had an appearance that is referred to as the "target" or a "donut" sign (Fig. 1).

Longitudinal images were obtained to confirm a bowel-within-bowel appearance. Color Doppler was used to evaluate the vascular structures of the bowel loops involved. Ultrasound guided hydrostatic reduction was performed in 31 of 34 patients. Three patients were not suitable for hydrostatic reduction due to contraindications and exclusion factors $(2,5)$. The contraindications in these patients were the age (a 2 month old baby), duration of symptoms (more than 48 hours) and ultrasound finding of ischemic bowel and Meckel diverticulitis.

In our clinical setup, the procedure, along with details of its benefits, probable outcome and possible complications, was explained to the parents or the legal guardian. Written informed consent was obtained and the surgical team informed of the procedure with a request to stand by in case of an emergency situation.

A common finding in the abdominal ultrasound exam was the presence of enlarged 


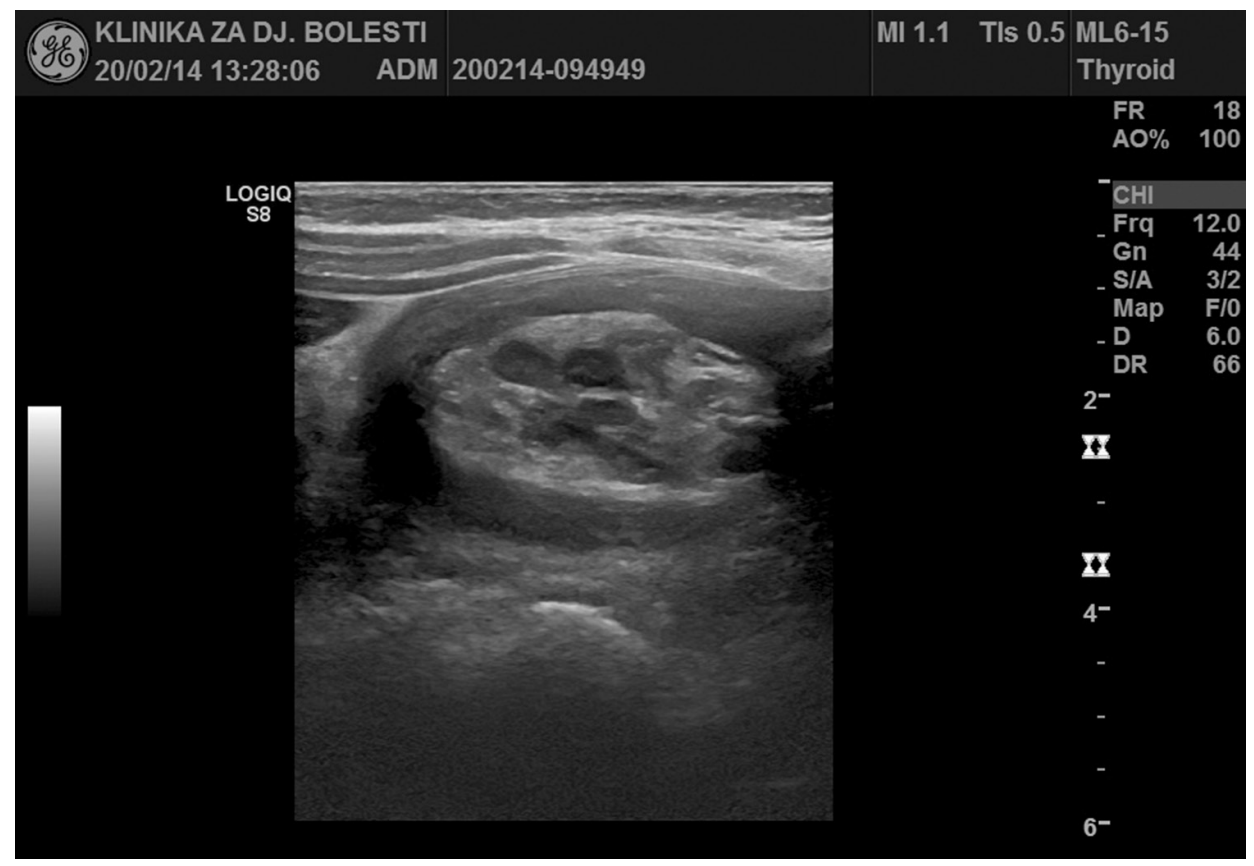

Fig. 1 Ultrasound exam. Viewed transversely, the alternating concentric hypoechoic and echogenic layers of an intussusception have an appearance that is commonly referred to as the "target" or "donut" sign.

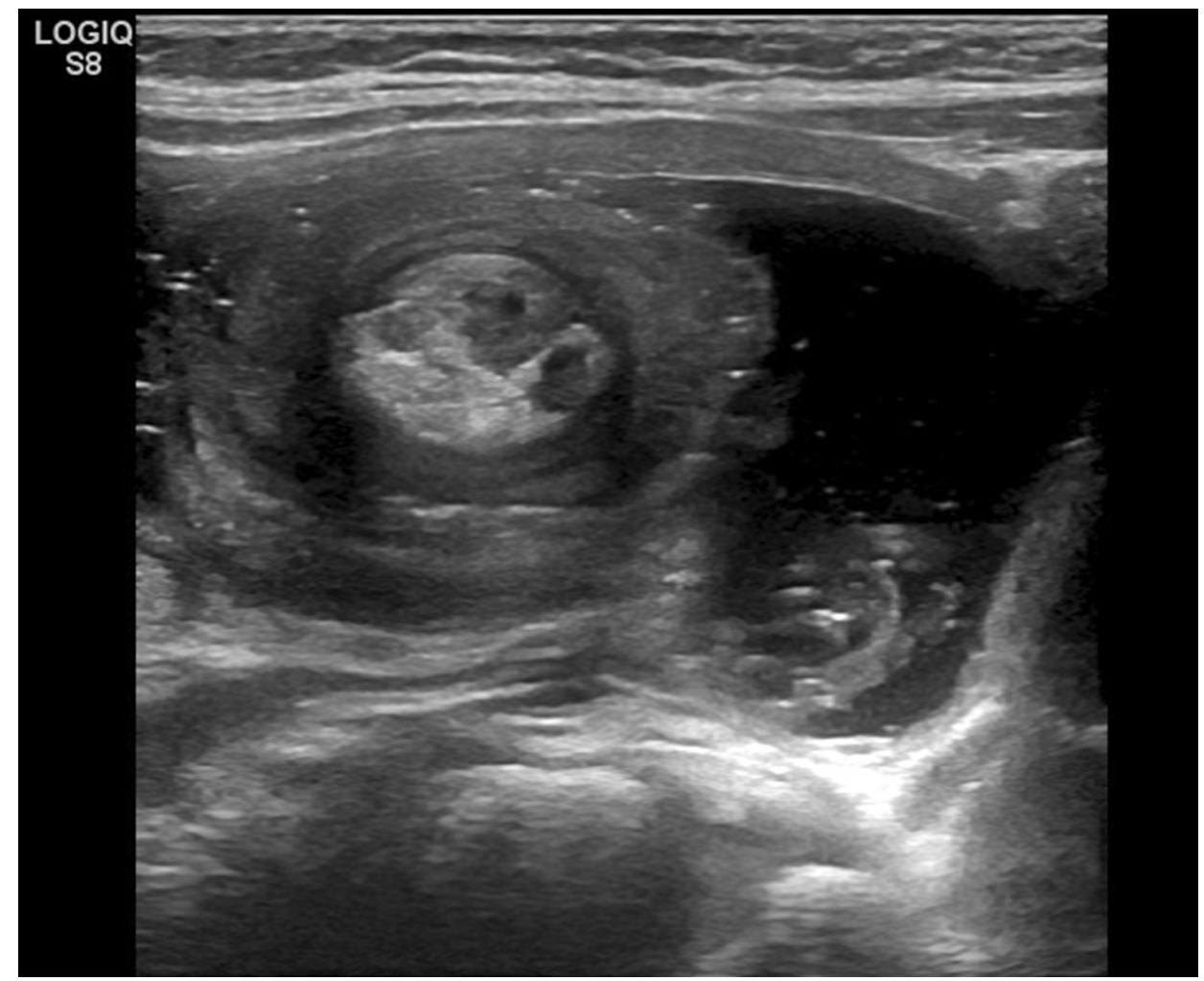

Fig. 2 Ultrasound exam. After initial application saline is traced in colon with visible intussusception. 

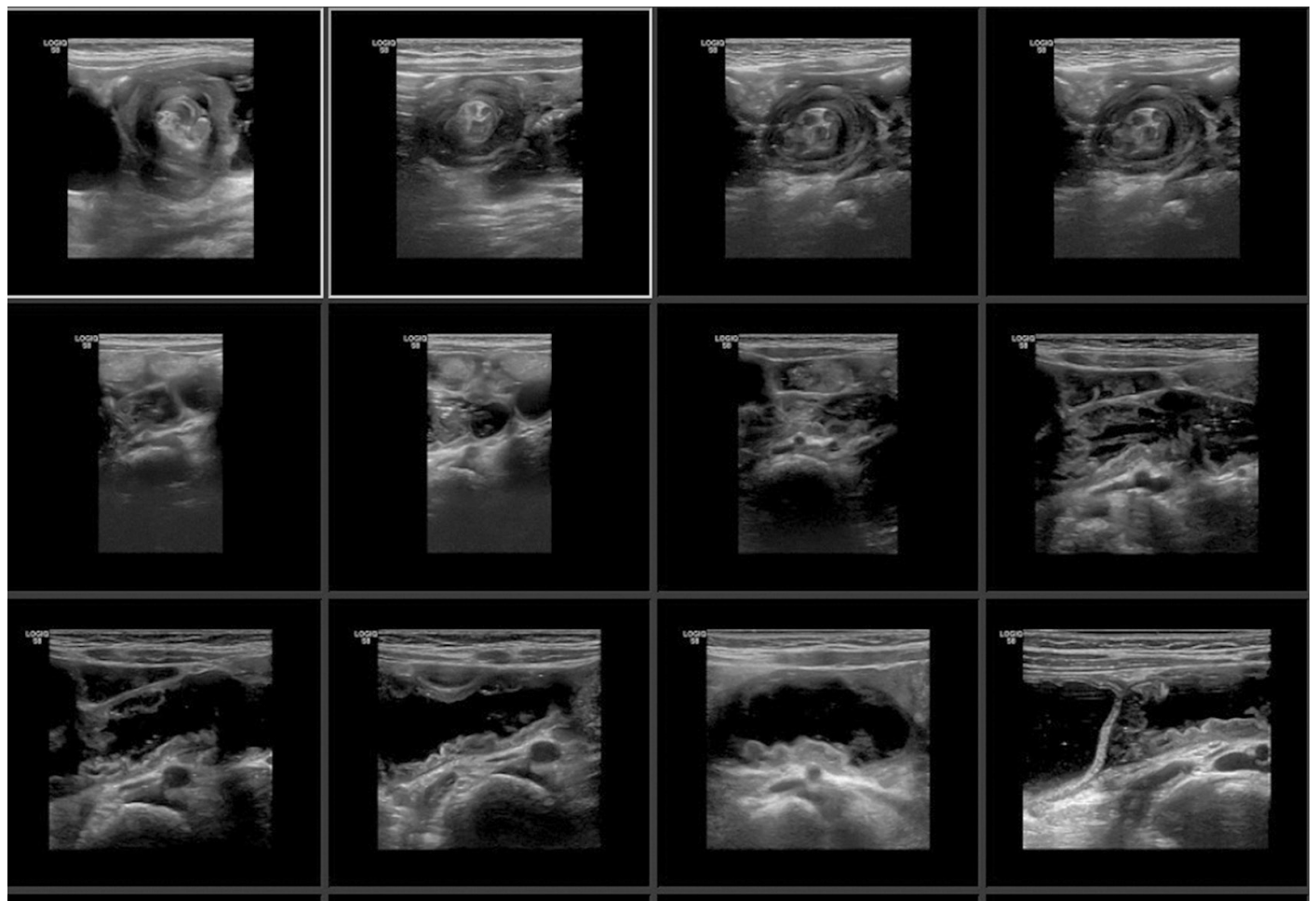

Fig. 3 Ultrasound exam. It is easy to follow reposition of intussusceptum through colon to the ileocecal valve.

mesenteric lymph nodes and hyperechoic mesentery that suggested inflammation. An age-appropriate Nelaton catheter was used. Based on our experience, the Nelaton catheter that should be used for the age group 0 to 2 is size 10 to $12 \mathrm{~F}$, for 3 to 5 years of age size 14 to $16 \mathrm{~F}$, and for 6 to 10 years size 18 to $20 \mathrm{~F}$, whereas for older children it should be size 22 F. The catheter is lubricated and placed into the rectum. When the catheter is introduced it is secured to the skin with tape. Using a pressure monitoring device, (pneumocolon) warm saline at body temperature is applied.

Distention of colon and the retrograde movement of the intussusceptum towards the cecum is monitored by ultrasound in real time (Fig. 2 and Fig. 3). Complete reduction is considered to be achieved once the edematous ileocecal valve is released and there is passage of saline through it into the ileum (Fig. 4 and Fig. 5). The Nelaton catheter is then removed.

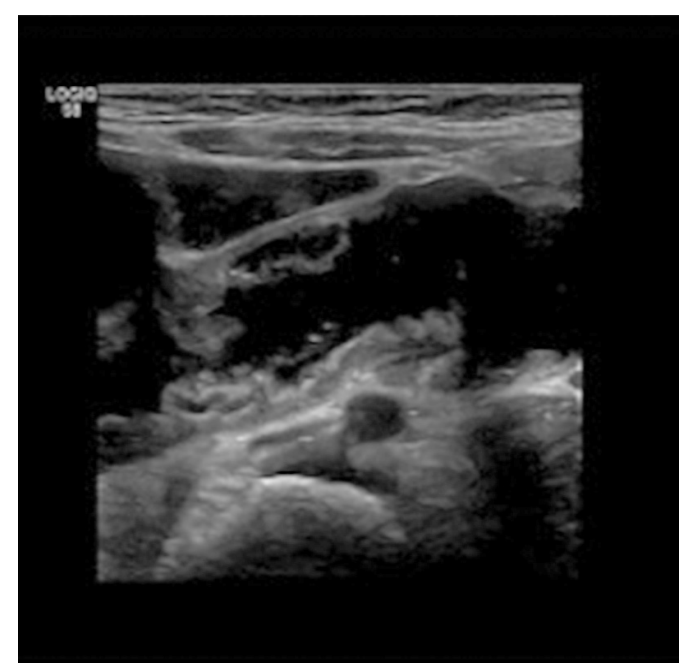

Fig. 4 Ultrasound exam. When intussusceptum crosses ileocecal valve the reposition is successful.

All patients were awake during the procedure. For saline enema dosage we used suggestions from Sarin et al. (15) for younger patients up to $500 \mathrm{ml}$ per procedure and for older ones up to $1000 \mathrm{ml}$ per procedure. 


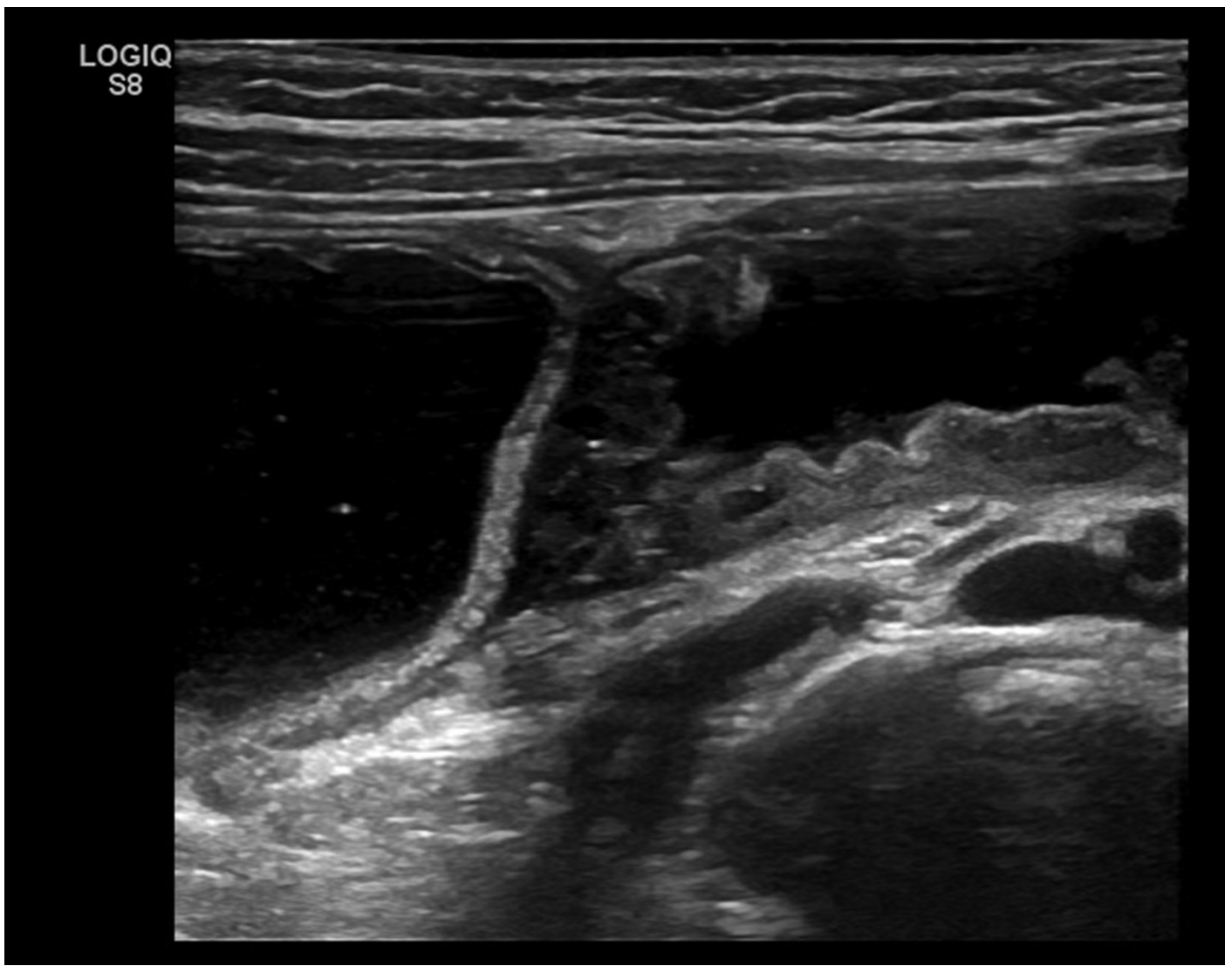

Fig. 5 Ultrasound exam. Successful reposition is confirmed when saline is seen in cecum and terminal part of ileum.

The clinical condition of the patient is monitored throughout the procedure. After the removal of the Nelaton catheter, all children passed stools immediately after the procedure. Six children vomited during or after the reduction and went to sleep. After the procedure the patient is transferred to the observation room under the supervision of a pediatric surgeon. A follow-up ultrasound scan is performed at 12 and 24 hours after the procedure (Fig. 6)

In three patients reinvagination was confirmed $24 \mathrm{~h}$ after the procedure. The main cause was the same "leading point" as in the first invagination - enlarged lymph nodes. Ultrasound guided hydrostatic reposition was repeated and was successful in all three patients. One patient had a second reinvagination in 48 hours and surgery was performed because of suspicion of ischemic necrosis of cecum due to Doppler signal loss within the cecal wall and free fluid in the pelvis. During surgical procedure viable cecum was found and reposition was performed. Short mesentery stalk was diagnosed as the probable cause of intussusception. The amount of saline used during the procedure ranged from 500 to $1200 \mathrm{ml}$. More than $1000 \mathrm{ml}$ of saline was used when most of the saline leaked out during the procedure. The average time taken from the inflow of saline into the rectum, up to the reduction of intussusception was 11 minutes. Minimal period of time for the procedure was 7 minutes, maximum 21 minutes. According to data from the literature it is possible to perform reposition two times in a row (15). We determined the maximal duration of the procedure at 30 minutes. 


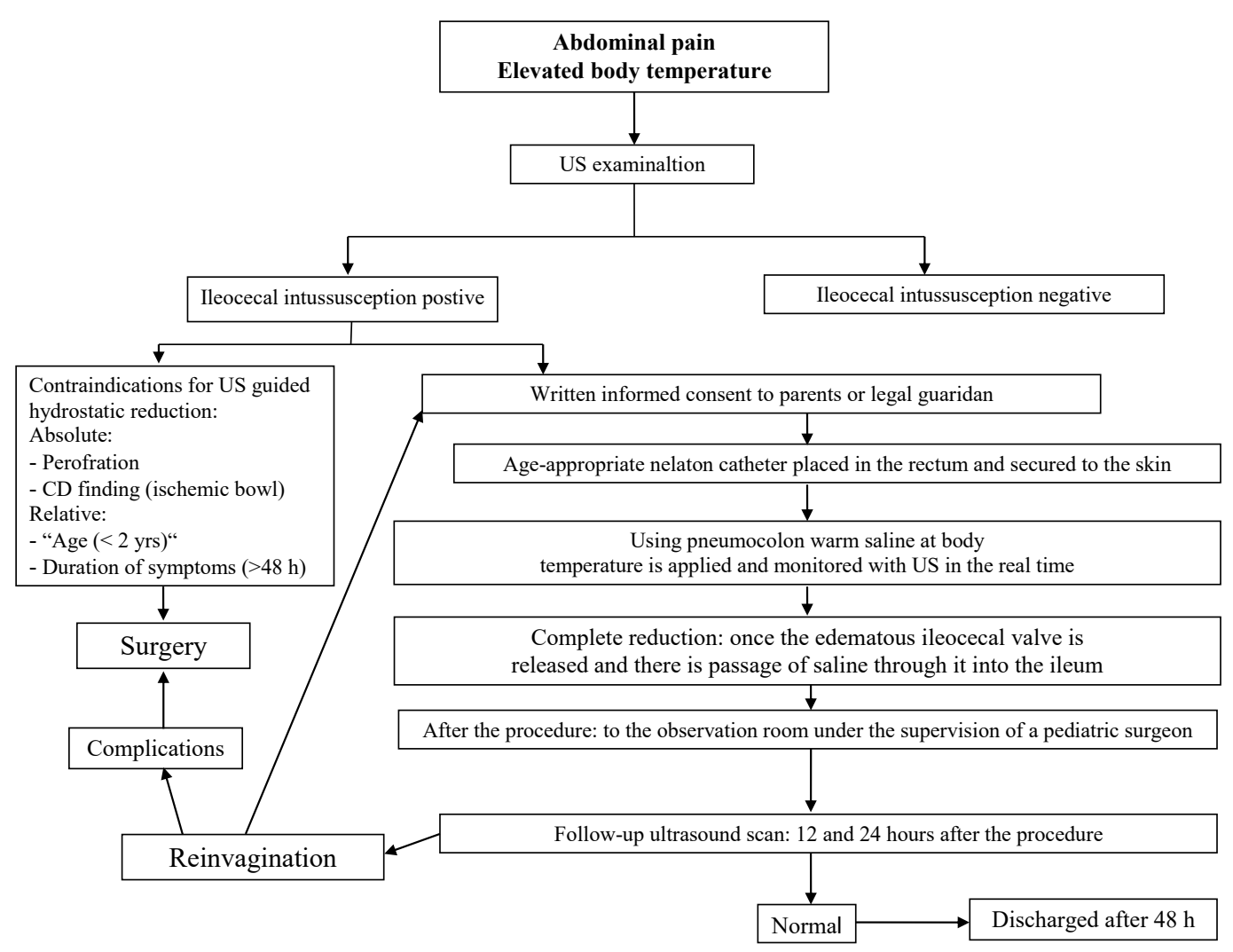

Fig. 6 Suggested algorithm of procedure for children with ileocecal intussusception.

\section{Results}

A total of 34 patients were confirmed as having ileocolic intussusception over the observed period of 2 years. Male predominance was observed, as previously described in literature. The youngest patient was 2 months and the oldest one was 13 years old. They all had intermittent colicky abdominal pain (16). After the diagnosis of ileocolic intussusception, three patients had contraindications for ultrasound guided hydrostatic reposition. Complete reduction of intussusception was achieved in the remaining 31 patients. In three patients, reinvagination occurred during the first 48 hours. In all patients ultrasound guided hydrostatic repositioning was successful. One patient had a second reinvagination at 48 hours and surgery was performed.
No patient suffered from perforation during hydrostatic reduction in this study. None of our patients who were suspected of developing a perforation showed radiographic evidence of pneumoperitoneum on plain abdominal X-rays. None of the patients suffered from rectal trauma. Most patients showed minimal free fluid in the peritoneum.

\section{Discussion}

Intussusceptions are classified as ileoileal, ileocolic and, in rare cases, colocolic intussusceptions. Intussusceptions may occur anywhere along the bowel, although in children there is a strong predilection for the ileocolic region (75\%-95\% cases). Ileocolic intussusceptions are the second most common and ileoileal and colocolic are uncommon (17). 
Ileoileal (Fig. 7) and colocolic intussusceptions are usually transient and, if smaller than $3 \mathrm{~cm}$, do not need any intervention.

Ileocolic intussusception is the second most common cause of acute intestinal obstruction in children. The most common cause is unknown. This group of children is believed to have idiopathic intussusception. There are three leading observations for explaining the possible etiology of idiopathic intussusception and the reasion why the Peyer patches are enlarged. The first suggests that often, the illness is preceded by an upper respiratory infection, the second that the ileocolic region has the highest concentration of lymph nodes in the mesentery, and the third that enlarged lymph nodes are often observed in patients who require surgery. Whether the enlarged Peyer patch is a reaction to the intussusception or the cause of it is unclear
(18). Rare causes include polyps, submucosal lipomas, Meckel's diverticulum and duplication cysts. The pathogenesis of idiopathic intussusception is not yet well established. It is believed to be secondary to an imbalance in the longitudinal forces along the intestinal wall. It is belived that the invaginating portion of the intestine completely enters into the receiving portion of the intestine. This process continues and more proximal areas follow, allowing the intussusceptum to proceed along the lumen of the intussuscipiens.

If the mesentery of the intussusceptum is lax and the progression is rapid, the intussusceptum can proceed to the distal colon or sigmoid and even prolapse out of the anus. The mesentery of the intussusceptum is invaginated with the intestine, leading to the classic pathophysiologic process of any bowel obstruction. Early in this process, lymphatic

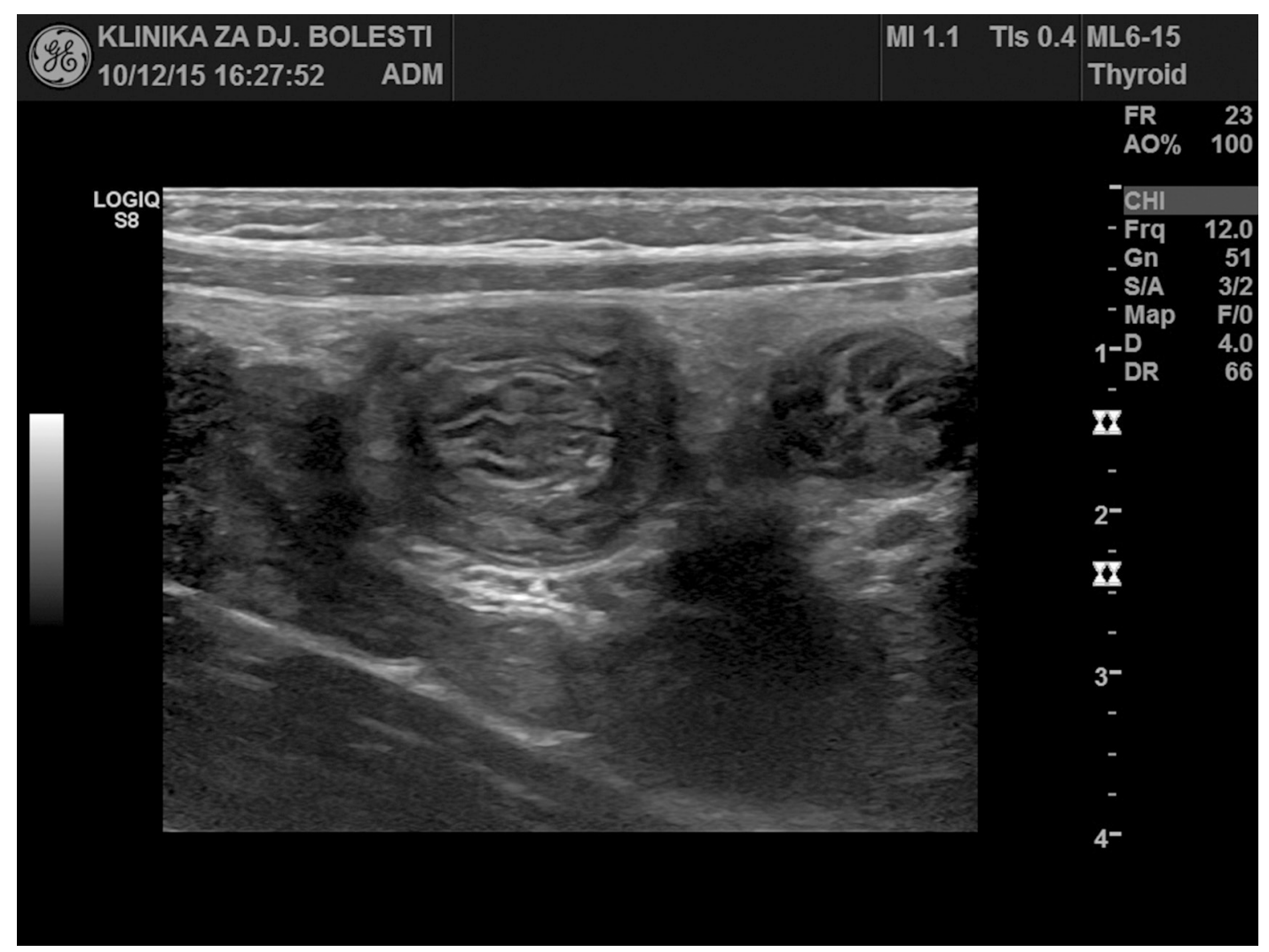

Fig. 7 Ileoileal invagination assessed by ultrasound. 
return is impeded; then, with increased pressure within the wall of the intussusceptum, venous drainage is impaired. If the obstructive process continues, the pressure reaches a point at which arterial inflow is inhibited, and infarction ensues. The intestinal mucosa is extremely sensitive to ischemia because it is farthest away from the arterial supply. Ischemic mucosa sloughs off, leading to the heme-positive stools and subsequently to the classic "currant jelly stool" (a mixture of sloughed mucosa, blood, and mucus). If untreated, transmural gangrene and perforation of the leading edge of the intussusceptum occur (18).

Symptoms include a sudden onset of intermittent colicky abdominal pain, drawing up of legs, vomiting, facial pallor, diarrhea, blood and mucus in the stools. Clinical examination may reveal a sausage shaped lump, with concavity towards the umbilicus and emptiness of the right iliac fossa (sign of Dance) $(3,5,13,19)$. If untreated, the bowel loop may become gangrenous, resulting in sloughing, perforation and peritonitis, and may eventually lead to death $(3,7,13,19)$.

In ileocolic intussusception, invagination of a bowel segment (the small bowel) into the lumen of the more distal bowel (the colon - cecum) occurs. The invaginated segment is carried distally by peristalsis. Mesentery, lymph nodes and vessels become involved with the intraluminal loop and compressed due to the position in the other colon loop. The "classic" clinical presentation is characterized by three manifestations: (a) acute colicky abdominal pain, (b) "currant jelly" or frankly bloody stools, and (c) either a palpable abdominal mass (2) or vomiting (1).

In radiology, classic abdominal $\mathrm{x}$-rays may demonstrate an elongated soft tissue mass (typically in the right upper quadrant in children) with bowel obstruction proximal to it (13). At that point, prior to ultrasound as a method, contrast enema is required.

After the application of contrast enema per rectum, intussusception as an occluding

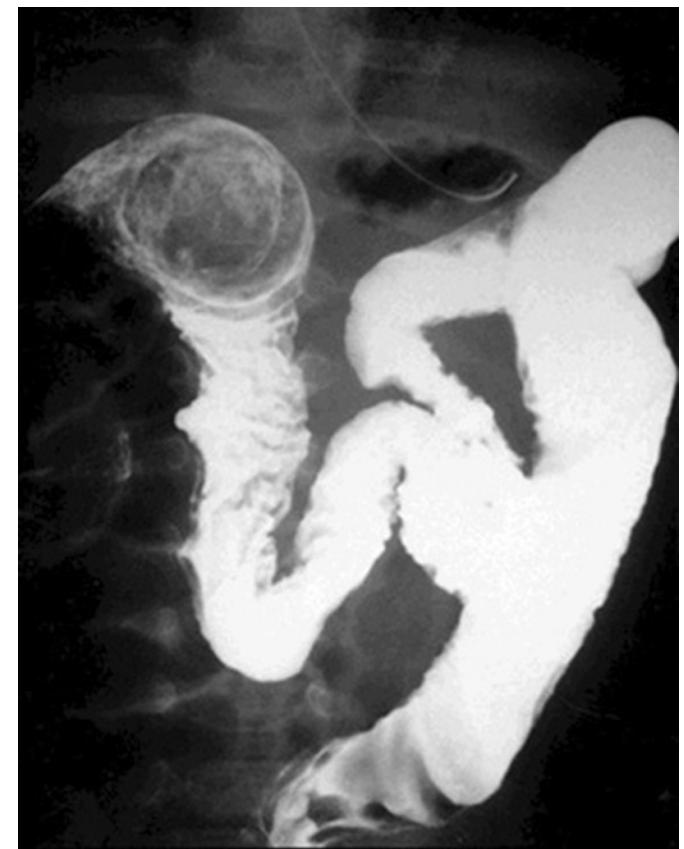

Fig. 8 Single contrast barium enema colonography. After application of contrast enema per rectum the intussusception as an occluding mass prolapsing into the lumen is presented, giving the "open fish mouth" appearance in upper right abdominal quadrant.

mass prolapsing into the lumen was presented, having the "open fish mouth" appearance (Fig. 8).

It is crucial to remember that intussusceptions may have recurrences following the initial nonsurgical reduction, especially in the first 72 hours. Reported recurrence rates, however, are highly variable, and are not calculated in a standardized manner. Most of the studies are based on relatively small trials. In addition, many of the reported recurrences are between $10 \%$ and 15\% (11). Ultrasound remains the best imaging modality for documenting recurrent intussusceptions, as well as ultrasound guided hydrostatic rereposition, while surgery is reserved for patients in whom nonsurgical reduction is contraindicated, where nonsurgical reduction fails, or in those with a suspected pathological lead point, as well as selected cases with several episodes of recurrence (7). 
In nonsurgical reduction therapeutic enemas include fluoroscopy guided hydrostatic (with barium or water-soluble contrast) and pneumatic (with air insufflations) reposition or ultrasound guided hydrostatic reposition. Surgical reduction traditionally includes access to the abdomen through a right periumbilical incision.

Ultrasound is a very useful examination for diagnosing intussusceptions, since it provides high sensitivity and specificity. In recent studies US had a sensitivity of $97.9 \%$, a specificity of $97.8 \%$, and a negative predictive value of $99.7 \%$ for intussusception (2, $3,5,12)$.

The 'target sign' described in literature is very useful and can be easily picked up by linear transducer (7.5-10 MHZ) (3, 7, 13, 19) even by an inexperienced radiologist. A small amount of free fluid in the peritoneum is common in most cases of intussusception $(2,3,5)$. Absence of blood flow in color Doppler studies of intussusception correlates significantly with irreducibility $(3,7$, 21, 22). We used color Doppler assessment in a majority of the patients and two of these patients showed absence of blood flow in the intussusceptions, so ultrasound guided hydrostatic reposition was not performed. The success rate is significantly lower for intussusception cases with the presence of bloody stool, free peritoneal fluid, and trapped fluid in the intussusception. The success rate is also lower when the intussusception is located in the left side of the abdomen (23).

Large lymph nodes (the cause for failure in one of the patients), trapped peritoneal fluid in the intussusception, and thick $(>10$ $\mathrm{mm}$ ) hypoechoic rim are the other predictors of irreducibility $(2,13)$. The absence of color flow within the intussusception, as the result of progressive constriction of mesenteric vessels by edematous loops, correlates with bowel necrosis and irreducibility $(3,20)$. Some other factors have been reported to decrease the chances of successful reduction: long duration of symptoms ( $>48$ hours), significant dehydration, radiographic evidence of small bowel obstruction, and patient age less than 3 months or more than 5 years $(2,5)$. A small amount of free fluid within the peritoneal cavity is a relatively common finding and does not affect reduction rates $(3,20)$.

In the presence of predictors of irreducibility, enema therapy can be tried, before resorting to surgery, if the clinical condition is favorable $(3,8,13,20)$. The attempt should be very gentle and careful. Nelaton catheter is used in the technique we use. This provides a relatively closed system for effective transmission of hydrostatic pressure, increasing the success rate.

There are several advantages for using ultrasound in the diagnosis and management of intussusception in children. It is simple, effective, economical, and less time-consuming, with fewer complications (very low perforation rate, $0.26 \%)(3,8)$. Only a short hospital stay is required (11). Also, examination is free of radiation hazard and is characterized by less morbidity (no incidence of pseudo-reduction, which is observed in fluoroscopic guided procedures). Another advantage is that in the case of perforation there is no possibility for chemical peritonitis as with Barium enema, no tension pneumoperitoneum as with air enema, no chance for fluid shifts as with hypertonic contrast medium solutions or tap water, and no mortality. If reinvagination occurs, the same method can be repeated $(20,24,25)$.

Plain radiograph of the abdomen should be obtained only where there is suspicion of complication (pneumoperitoneum). Ultrasound follow up is required 12 hours and 24 hours after the treatment. Cooperation with a pediatric surgeon is essential during the whole procedure.

Conditions that can mimic ileocolic intussusception are occasionally seen in chil- 
dren with abdominal pain. Thickening of the cecum or ascending colon due to an inflammatory or infectious process can occasionally produce an appearance at ultrasound that resembles the target sign of intussusception. Small bowel intussusceptions are sometimes encountered at ultrasound and, like ileocolic intussusceptions, may have a target or donut appearance, but are transient, often subsiding during examination However, these small bowel intussusceptions are typically smaller in diameter than ileocolic intussusceptions, are more centrally located within the abdomen, and are often incidental findings. Those that are less than $3.5 \mathrm{~cm}$ in length are usually asymptomatic and may reduce spontaneously within minutes $(7,26)$.

\section{Conclusion}

Ultrasound guided hydrostatic reduction of ileocolic invagination in children, using warm saline at body temperature, is a very simple and effective technique. The main advantages are that it is less time consuming in comparison to surgery, more cost effective, there is no radiation hazard (which complies with the ALARA principles), involves minimal complications and short hospital stay (27). The procedure has a high success rate in properly selected patients. In our institution, the success rate was $91.2 \%$ and the recurrence rate was $8.8 \%$. Following our experience, we strongly advise that this procedure be advocated in detected intussusception and selected patients, with a view to avoiding radiation and unwarranted surgeries.

Authors' contributions: Conception and design: ATB and TG; Acquisition, analysis and interpretation of data: ATB and TG; Drafting the article: GR; Revising the article critically for intellectual content: GR, ATB and TG; Approved final version of the manuscript: GR.

Conflict of interest: The authors declare that they have no conflict of interest.

\section{References}

1. Khorana J, Singhavejsakul J, Ukarapol N, Laohapensang $\mathrm{M}$, Wakhanrittee J, Patumanond J. Enema reduction of intussusception: The succsess rate of hydrostatic and pneumonic reduction. Therapeutics and Clinical Risk Management. 2015;11:1837-42.

2. del-Pozo G, Albillos JC, Tejedor D, Calero R, Rasero $\mathrm{M}$, de-la-Calle $\mathrm{U}$, et al. Intussusception in children: current concepts in diagnosis and enema reduction. Radiographics. 1999;19(2):299-319.

3. Cogley JR, O’Connor SC, Houshyar R, Al Dulaimy K. Emergent pediatric US: What every radiologist should know. Radiographics. 2012;32(3):65165.

4. Vasavada P. Ultrasound evaluation of acute abdominal emergencies in infants and children. Radiol Clin North Am. 2004;42(2):445-56.

5. Medscape.com [homepage on the Internet]. New York: Blanco FC, Wilkes G, King L, Chahine AA. Windle ML, Li Buk, et al. Intussusception. [updated 2016 March 16; cited 2016 May 9]. Available from: http://emedicine.medscape.com/ article/930708-overview.

6. Marc CW. Intestinal Obstruction. In: Russel RG, Williams NS, Bulstrode CJK, editors. Bailey \& Love's Short Practice of Surgery. London: Arnold Publishers; 2000. p. 1067-106.

7. Niramis R, Watanatittan S, Kruatrachue A, Anuntkosol M, Buranakitjaroen V, Rattanasuwan T, et al. Management of recurrent intussusception: nonoperative or operative reduction? J Pediatr Surg. 2010;45(11):2175-80.

8. Shastri MD, Rucha S, Eke D. Ultrasound guided reduction of an ileocolic intussusception by a hydrostatic method by using normal saline enema in pediatric patients: A study of 30 cases. J Clin Diagn Res. 2012;6(10):1722-25.

9. DanemanA, Navarro O, Intussusception I. A review of diagnostic approaches. Pediatr. Radiol. 2003;33(2):79-85.

10. Applegate KE. Clinically suspected intussusception in children: evidence-based review and self-assessment module. AJR Am J Roentgenol. 2005;185(Suppl 3):S175-S183.

11. Gray MP, Li SH, Hoffmann RG, Gorelick M. Recurrence rates after intussusception enema reduction: a meta-analysis. Pediatrics. 2014;134(1):1109. 
12. HryhorczukA L, Strouse PJ. Validation of US as a first-line diagnostic test for assessment of pediatric ileocolic intussusception. Pediatr Radiol. 2009;39(10):1075-9.

13. Radiopaedia.org [homepage on the Internet]. Kang O, Amini B. Intussusception. [cited 2016 May 9]. Available from: http://radiopaedia.org/ articles/intussusception.

14. Kim YG. Diagnosis and treatment of childhood intussusception using real time ultrasonography and saline enema. Preliminary report. J Korean Soc Med Ultrasound. 1982;1:66-70.

15. Sarin YK, Rao JS, Stephen E. Ultrasound guided water enema for hydrostatic reduction of childhood intussusception-a preliminary experience. Indian J Radiol Imaging. 1999;9:59-63.

16. Ogundoyin OO, Olulana DI, Lawal TA. Childhood intussusception: A prospective study of management trend in a developing country. Afr J Paediatr Surg. 2015;12(4):217-20.

17. Choi SH, Han JK, Kim SH, Lee MJ, Lee KH et al. Intussusception in adults: from stomach to rectum. AJR Am J Roentgenol. 2004;183 (3):691-8.

18. Vujović D, Lukać M, Sretenović A, Krstajić T, Ljubić V, Antunović SS. Indications for repeated enema reduction of intussusception in children. Srp Arh Celok Lek. 2014;142(5-6):320-4.

19. Ugwu BT, Legbo JN, Dakum NK, Yiltok SJ, Mbah N, Uba FA. Child hood intussusception: 9 year review. Ann Trop Paediatr. 2000;20(2):131-5.

20. Mirilas P, Koumanidou C, Vakaki M, Skandalakis P, Antypas S, Kakavakis K. Sonographic features indicative of hydrostatic reducibility of intestinal intussusception in infancy and early childhood. Eur Radiol. 2001;1112):2576-80.

21. Sanchez TRS, Potnick A, Graf JL, Abramson LP, Patel CV. Sonographically Guided Enema for Intussusception Reduction: A Safer Alternative to Fluoroscopy. J Ultrasound Med. 2012;31:1505-8.

22. Lim HK, Bae SH, Lee KH, Seo GS, Yoon GS. Assessment of reducibility of ileocolic intussusception in children: Usefulness of color Doppler sonography. Radiology. 1994;191:781-5.

23. He N, Zhang S, Ye X, Zhu X, Zhao Z, Sui X. Risk factors associated with failed sonographically guided saline hydrostatic intussusception reduction in children. J Ultrasound Med. 2014;33(9):1669-75.

24. Navarro OM, Daneman A, Chae A. Intussusception: The use of delayed, repeated reduction attempts and the management of intussusception due to pathologic lead points in pediatric patients. AJR. 2004;182:1169-76.

25. Daneman A, Navarro O. Intussusception, Part 2: An Update on the evolution of management. Pediatr Radiol. 2004:34:97-108.

26. Munden MM, Bruzzi JF, Coley BD, Munden RF. Sonography of pediatric small-bowel intussusception: differentiating surgical from nonsurgical cases. AJR Am J Roentgenol. 2007;188(1):275-9.

27. Sanchez TR, Doskocil B, Stein-Wexler R. Nonsurgical management of childhood intussusception: retrospective comparison between sonographic and fluoroscopic guidance. J Ultrasound Med. 2015;34(1):59-63. 